\title{
Determinação do índice de rigor-mortis e sua relação com a degradação dos nucleotídeos em tambaqui (Colossoma macropomum), de piscicultura e conservados em gelo
}

\author{
Determination of the rigor-mortis index and its relation with rate decrease \\ nucleotides of a cultivated amazonian fish
}

\author{
Neiva Maria de Almeida ${ }^{1}$ Gilvan Machado Batista ${ }^{2}$ \\ Makie Kodaira ${ }^{3}$ Adalberto Luís Val ${ }^{4}$ Edson Lessi ${ }^{5}$
}

\section{RESUMO}

Este trabalho teve a finalidade de determinar o índice de rigor-mortis e sua relação com a taxa de degradação dos nucleotídeos do tambaqui (Colossoma macropomum) cultivado e conservado em gelo. O início da rigidez (IR) muscular para o pescado ocorreu 20 minutos $(I R=80,1 \%)$ após a morte por asfixia entre camadas de gelo. A contração total ocorreu aos 30 minutos (IR=99,4\%) e a resolução total do rigor cerca de 360 horas (15 dias). A concentração de ATP diminuiu cerca de $50 \%$ aos 20 minutos. Aos 30 minutos após a morte, ainda restavam aproximadamente $30 \%$ da quantidade inicial de ATP. Somente após duas horas, o teor de ATP se aproximou de zero, indicando que os exemplares submetidos ao resfriamento com gelo apresentaram o fenômeno de "cold shock " e não rigor-mortis. O valor $k$ mostrou pequenas variações até cerca de 2 horas após a morte e, em seguida, um aumento progressivo, variando de $0,7 \%$, a $11,5 \%$ no $9^{\circ}$ dia de estocagem.

Palavras-chave: Colossoma macropomum, piscicultura, conservação em gelo, nucleotídeos, rigormortis.

\section{ABSTRACT}

This study examined the rigor index and its relation with rate decrease nucleotides of a cultivated Amazonian fish, Colossoma macropomum, stored in ice. The rigor index was detected 20 minutes $(I R=80.1 \%)$ after death in ice. Total muscle contraction occurred after 30 minutes
(IR=99.4\%) and total resolution of rigor after 15 days in ice. ATP concentrations decreased after $20 \mathrm{~min}$ to about half, 50\%; after 30 minutes residual ATP was $30 \%$ and after 2 hours practically disappeared. This suggests that the $\mathbf{C}$. macropomum specimens stored in ice presented the cold shock phenomenon rather than rigor-mortis. The $K$ value varied little up to 2 hours after death and then increased progressively up to $11.5 \%$ on the 9 th day of storage.

Key words: Colossoma macropomum, pisciculture, store in ice, nucleotides, rigor-mortis.

\section{INTRODUÇÃO}

Dados da Food and Agriculture Organization (FAO) mostraram que a produção de pesca da captura se manteve estável e que o aumento de 20 milhões de toneladas em 1999, se deveu, sobretudo ao crescimento da aquicultura, sendo este o sistema de produção de alimentos que mais cresce no mundo, com uma taxa de $2 \%$ ao ano (FAO, 2000).

A região Norte possui a maior diversidade de peixes de água doce com mais de 1500 espécies já descritas (JUNK et al., 1997). Nesta região, a pesca é baseada na atividade extrativista, o que tem contribuído para colocar em perigo de extinção algumas

${ }^{1}$ Engenheiro de Pesca, Doutor, Universidade Federal do Amazonas, Rua Alexandre Amorim 330, 69010-300, Manaus, Amazonas, Brasil. E-mail:neivaa@yahoo.com. Autor para correspondência.

${ }^{2}$ Engenheiro de Pesca, Mestre, Universidade Federal do Amazonas (UFAM), Brasil.

${ }^{3}$ Biólogo, Doutor, Universidad Central da Venezuela (UVC), Caracas, Venezuela.

${ }^{4}$ Biólogo, Doutor, Instituto Nacional de Pesquisa da Amazônia (INPA), Brasil.

${ }^{5}$ Farmacêutico, Doutor, Instituto Nacional de Pesquisa da Amazônia (INPA), Brasil. 
espécies capturadas na região, fator determinante que levou o Governo Federal a adotar medidas regulamentares de proteção às espécies de peixes na Amazônia (MMA, IBAMA, 1997).

A participação do tambaqui na produção extrativista vem diminuindo ao longo dos anos, registrando somente 1.849 toneladas em 1998 (MMA, IBAMA, CEPENE, 2000). Segundo GRAEF (1995), o tambaqui é a espécie nativa que mais tem despertado interesse para a piscicultura no estado do Amazonas, devido à preferência do consumidor, excelente preço de mercado e maior conhecimento sobre a biologia e desempenho em cativeiro.

A comercialização e o transporte são etapas de destaque para o sucesso da piscicultura, uma vez que o pescado é altamente perecível e, imediatamente após a morte, sofre uma série de reações autolíticas no músculo, que influenciam suas características organolépticas originais. Os primeiros processos autolíticos no tecido muscular envolvem mudanças dos nucleotídeos e carboidratos (BERAQUET \& LINDO, 1985).

As discussões sobre as análises de nucleotídeos e suas alterações durante a estocagem têm sido pesquisadas. As mudanças autolíticas ocorrem nos peixes através de dois tipos de deterioração; enzimática e bacteriológica. As atividades enzimáticas individuais que participam da reação mediante as quais o ATP se degrada a hipoxantina, através de IMP e da inosina varia muito de espécie para espécie, depende da temperatura e do pH e também de outros fatores como as condições de manipulação, com o estado físico dos peixes antes de capturá-los, dos métodos de captura e da maneira como são sacrificados (AMLACHER, 1965; HUSS, 1988). O rigor aparece em conseqüência das primeiras mudanças bioquímicas post-mortem, e pode ser influenciado por fatores extrínsecos, como a captura, a temperatura de estocagem e, principalmente, pela maneira como o peixe é sacrificado (BOYD et al., 1984).

O estudo de rigor-mortis está geralmente relacionado aos efeitos das temperaturas de estocagem, especialmente em peixes tropicais. Com respeito a este ponto, as experiências mostram a ocorrência do "cold shock", termo que tem sido usado para descrever um provável rigor que ocorre em algumas espécies de peixes tropicais. Apesar de se usarem as mesmas explicações para os mecanismos entre o "cold shock" e rigor, NAMBUDIRI \& GOPAKUMAR (1988) estudaram peixes tropicais e mostraram que estes fenômenos apresentam diferenças principalmente no que se refere ao conteúdo de ATP presente no músculo no momento que se estabelece a rigidez muscular e observaram que o estado de "cold shock" se estabeleceu quando $40 \%$ da concentração de ATP ainda permanecia no músculo.

Este trabalho tem o objetivo de determinar o índice de rigor-mortis e sua relação com a taxa de degradação dos nucleotídeos e determinar o valor K, que é utilizado para medir o frescor do peixe baseado nas alterações autolíticas, relacionando a concentração de inosina e hipoxantina e o conteúdo total de compostos obtidos da degradação de ATP, no músculo dorsal do tambaqui produzido pela piscicultura e conservado em gelo, para verificar se estes parâmetros serão considerados como índice para avaliação do frescor do pescado.

\section{MATERIAL E MÉTODOS}

O tambaqui procedente dos tanques de engorda do "Sítio dos Gaúchos" - Manaus, Am, foi capturado com rede de arrasto. Foram utilizados 7 exemplares, com peso médio de $2,5 \mathrm{~kg}$ e comprimento médio de $47,9 \mathrm{~cm}$ para determinação do índice de rigormortis no pescado e para a determinação do ATP e dos seus produtos de degradação (valor K) no músculo dorsal.

Os peixes foram analisados quanto ao tempo de entrada em rigor-mortis, avaliados desde a captura até a resolução do rigor. O rigor dos exemplares foi medido imediatamente após o sacrifício por asfixia entre camadas de gelo, na proporção de 1:1, e com 10, 20 e 30 minutos após a morte, com posteriores medidas realizadas diariamente, procurando manter um período de 24 horas, até 15 dias de estocagem. O índice de rigor foi medido segundo com BITO et al. (1983), e calculado de acordo com a equação:

$$
\mathrm{IR}=\frac{\mathrm{Do}-\mathrm{D}}{\text { Do }} \times 100
$$

Onde: Do = valor da distância que separa a base da nadadeira caudal ao ponto de referência, imediatamente após a morte e $\mathrm{D}=$ valor da distância que separa a base da nadadeira caudal ao ponto de referência nos intervalos de tempos selecionados.

As concentrações de ATP e seus produtos degradados ADP, AMP, IMP, HxR e Hx, foram determinadas no músculo dorsal do tambaqui, de acordo com a metodologia descrita por SCHULTE et al. (1992), modificado por VAL et al. (1994). Realizou-se amostragem nos exemplares imediatamente após o sacrifício por asfixia entre camadas de gelo, com 20, 80 e 120 minutos da medida inicial e congelou-se em nitrogênio líquido até o transporte para o Centro de Pesquisa em Tecnologia de Alimento (CPTA), do Instituto de Pesquisa da Amazônia (INPA). Foram realizadas 
amostragens até 12 dias de estocagem. Coletaram-se $200 \mu \mathrm{g}$ do músculo da parte dorsal aos quais foram adicionados $400 \mu 1$ de ácido perclórico a $8 \%$ (v/v). Em seguida, eles foram homogeneizados e centrifugados a $3000 \mathrm{rpm}$ por 7 minutos e $200 \mu 1$ do sobrenadante foi neutralizado com $23 \mu \mathrm{l}$ de $\mathrm{KOH}(6 \mathrm{M})$. Em seguida, foi realizada Cromatografia Líquida de Alta Eficiência (CLAE), utilizando cromatógrafo modelo 2152, com bomba de titânio 2150 acoplada a um integrador LKB modelo 2220. Nas separações, utilizou-se uma coluna de troca iônica Aquapore Ax 300, de $7 \mathrm{~mm}$ mantida a $55^{\circ} \mathrm{C}$ com um fluxo de $2 \mathrm{~mL} / \mathrm{min}^{-1}$. Os tampões de eluição foram preparados com reagentes de alto grau analítico (Sigma), filtrados e pré-purificados usando a coluna econo Bio Rad. A eluição foi feita com solução tampão fosfato $60 \mathrm{mmol} / \mathrm{L} \mathrm{KH}_{2} \mathrm{PO}_{4}, \mathrm{pH} \mathrm{3,2}$, para os cinco primeiros minutos, seguindo um gradiente linear até $750 \mathrm{mM} \mathrm{KH}_{2} \mathrm{PO}_{4} \mathrm{pH} \mathrm{3,5}$ durante 9 minutos. Estas condições foram mantidas durante 15 minutos. Em seguida, o sistema foi lavado com solução tampão inicial durante cinco minutos para reequilibrar a coluna antes da segunda corrida. Soluções padrões de AMP, ADP IMP, HxR, Hx, $(50 \mu \mathrm{g})$ e ATP $(100 \mu \mathrm{g})$ foram preparadas e usadas para determinar o tempo de retenção e construir as curvas padrões, abrangendo um intervalo de concentração relevante ao planejado. As faixas de concentração foram compreendidas entre 0,003 a 1,0 $\mu \mathrm{g}$. As concentrações de todas as soluções padrões foram duplamente testadas através de espectrofotometria. $\mathrm{O}$ volume exato a ser injetado na coluna variou de acordo com a melhor visualização dos picos $(25,50$ e $100 \mu 1)$.

$\mathrm{O}$ valor $\mathrm{K}$ foi obtido pelas concentrações dos nucleotídeos, é definido segundo SAITO et al. (1959), pela equação:

$K=\frac{H \times R+H x}{A T P+A D P+A M P+I M P+H \times R+H x} \times 100$

Onde: $\mathrm{ATP}=$ Adenosina trifosfato; $\mathrm{ADP}=$ Adenosina difosfato; $\mathrm{AMP}=$ Adenosina monofosfato; IMP = Inosina monofosfato; HxR = Inosina; $\mathrm{Hx}=$ Hipoxantina

A análise estatística foi realizada através de ajuste de regressão linear simples conforme ZAR (1996).

\section{RESULTADOS E DISCUSSÃO}

Existem inúmeros modos de sacrificar o peixe; por asfixia em gelo, anestesiados em gelo e água e ou por destruição do cérebro. O método usado para sacrificar o peixe pode acelerar ou atrasar o início do rigor. O sacrifício por destruição do cérebro, também conhecido como "iki jime", permite manter alto nível de ATP no músculo (BOYD et al., 1984). Logo após a morte por asfixia entre camadas de gelo, não houve contração muscular. O progresso do rigor-mortis (Figura 1) ocorreu linearmente até 20 minutos após a morte e o índice de rigor foi de $80,1 \%$. Aos 30 minutos, ocorreu o máximo de contração $(99,4 \%)$. O início da resolução do rigor ocorreu após 144 horas (6 dias), com o índice de rigor de $91,5 \%$, e a resolução total com 360 horas, (15 dias) apresentando o índice de rigor de $46,7 \%$.

Sabe-se que o estabelecimento do rigormortis é conseqüência direta da concentração de ATP. Convém mencionar que, quando a concentração de ATP é menor que $10^{-4} \mu \mathrm{mol} \mathrm{g} \mathrm{g}^{-1}$, todas as reservas de ATP e de fosfato de creatina tem se esgotado, o que produz uma rigidez das fibras musculares, com o conseqüente aparecimento do rigor-mortis (RABELO, 1988).

A concentração de ATP do músculo de tambaqui (Figura 2) decresceu aproximadamente 50\%, aos 20 minutos, após a morte do tambaqui por asfixia, entre camadas de gelo, momento em que se deu a contração inicial com índice de rigor de $80,1 \%$. Aos 30 minutos, quando ocorreu a contração total ( $I R=99,4 \%)$ ainda restava no músculo aproximadamente $30 \%$ da quantidade inicial de ATP. Aos 80 minutos, o músculo ainda possuía aproximadamente $20 \%$ da concentração inicial de ATP. Somente após 2 horas, o teor de ATP se aproximou de zero.

NAMBUDIRI \& GOPAKUMAR (1988) observaram que o fenômeno de "cold shock" difere do rigor-mortis em função do conteúdo de ATP no músculo do pescado. Estes autores afirmaram que, em peixes tropicais, o fenômeno de "cold shock", ocorreu quando ainda havia $40 \%$ do ATP nos músculos dos peixes. PEREZ et al. (2001) relataram que o híbrido de "cachama" cultivado e sacrificado em gelo $\left(0^{\circ} \mathrm{C}\right)$ sofreu uma contração violenta conhecida como "cold shock" e que, nesse momento, o conteúdo de ATP foi de $0,50 \mu \mathrm{mol} / \mathrm{g}$ no músculo. CURRAN et al. (1986) estudaram as reações de "cold shock” em tilápia, (Oreochromis aureus/niloticus), e quando sacrificada a $0^{\circ} \mathrm{C}$ esta apresentou reação de "cold shock", com início da rigidez 2 horas depois de colocada em gelo e rigidez total depois de 8 horas. PARRY et al, (1987) estudaram o efeito do "cold shock" em carpa chinesa (Aristychthys nobilis) e em truta arco-íris (Salmo gairdnerii) sacrificadas e estocadas à temperatura de $0^{\circ} \mathrm{C}$. A carpa sofreu $\mathrm{o}$ fenômeno de "cold shock", enquanto a truta não sofreu esse fenômeno.

Ciência Rural, v.35, n.3, mai-jun, 2005. 


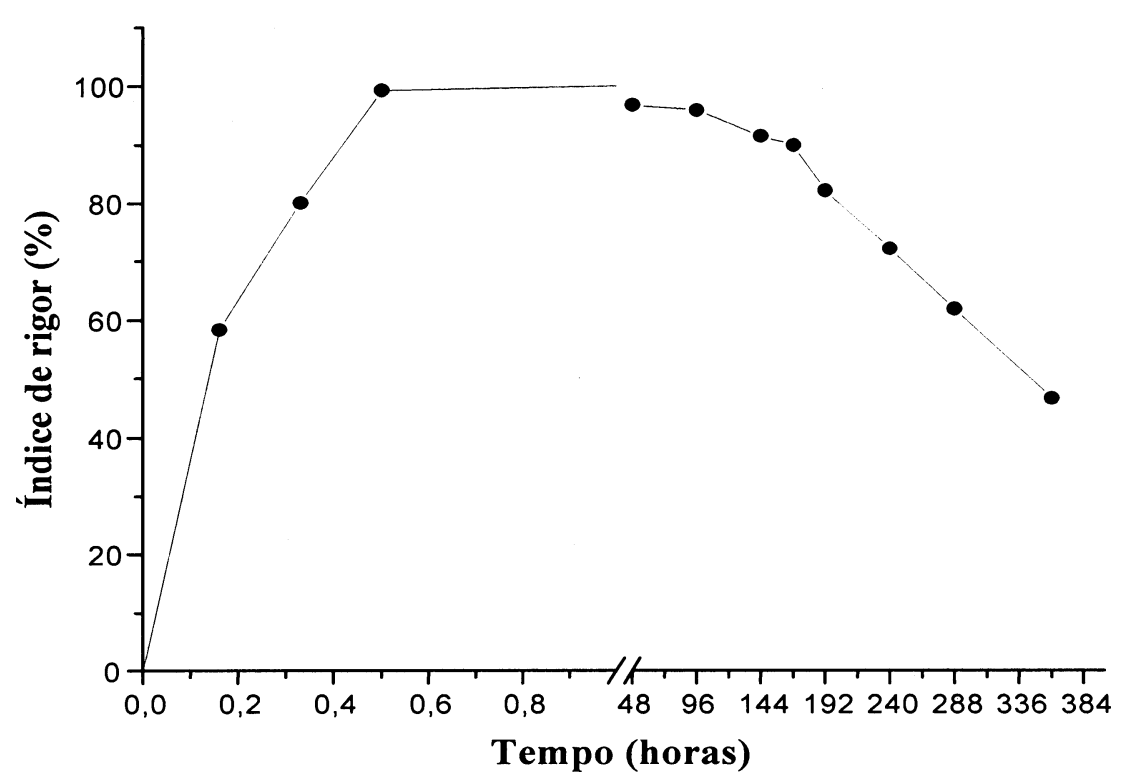

Figura 1 - Progresso do rigor-mortis (\%) do tambaqui, cultivado e estocado em gelo.

As concentrações iniciais de ATP e ADP no músculo dorsal de tambaqui cultivado foram similares, provavelmente devido à resíntese do ATP via fosfato de creatina (Figura 2). Aos 20 minutos, houve um decréscimo de cerca de $50 \%$ do valor inicial de ATP, e o ADP aumentou praticamente o triplo do valor inicial, paralelamente ocorreu acúmulo de AMP.
Aos 80 minutos após o sacrifício, os níveis de ATP $(0,04 \mathrm{mmol} / \mathrm{g})$ e $\operatorname{ADP}(0,38 \mathrm{mmol} / \mathrm{g})$ decresceram. Os níveis de ATP diminuíram $80 \%$ em relação aos valores iniciais e mostraram um residual de $6,13 \%$ após 2 horas. Os teores de ADP permaneceram altos, 0,56 e $0,31 \mathrm{mmol} / \mathrm{g}$ com 20 e 1728 minutos respectivamente, possivelmente interferindo nos níveis de IMP e das

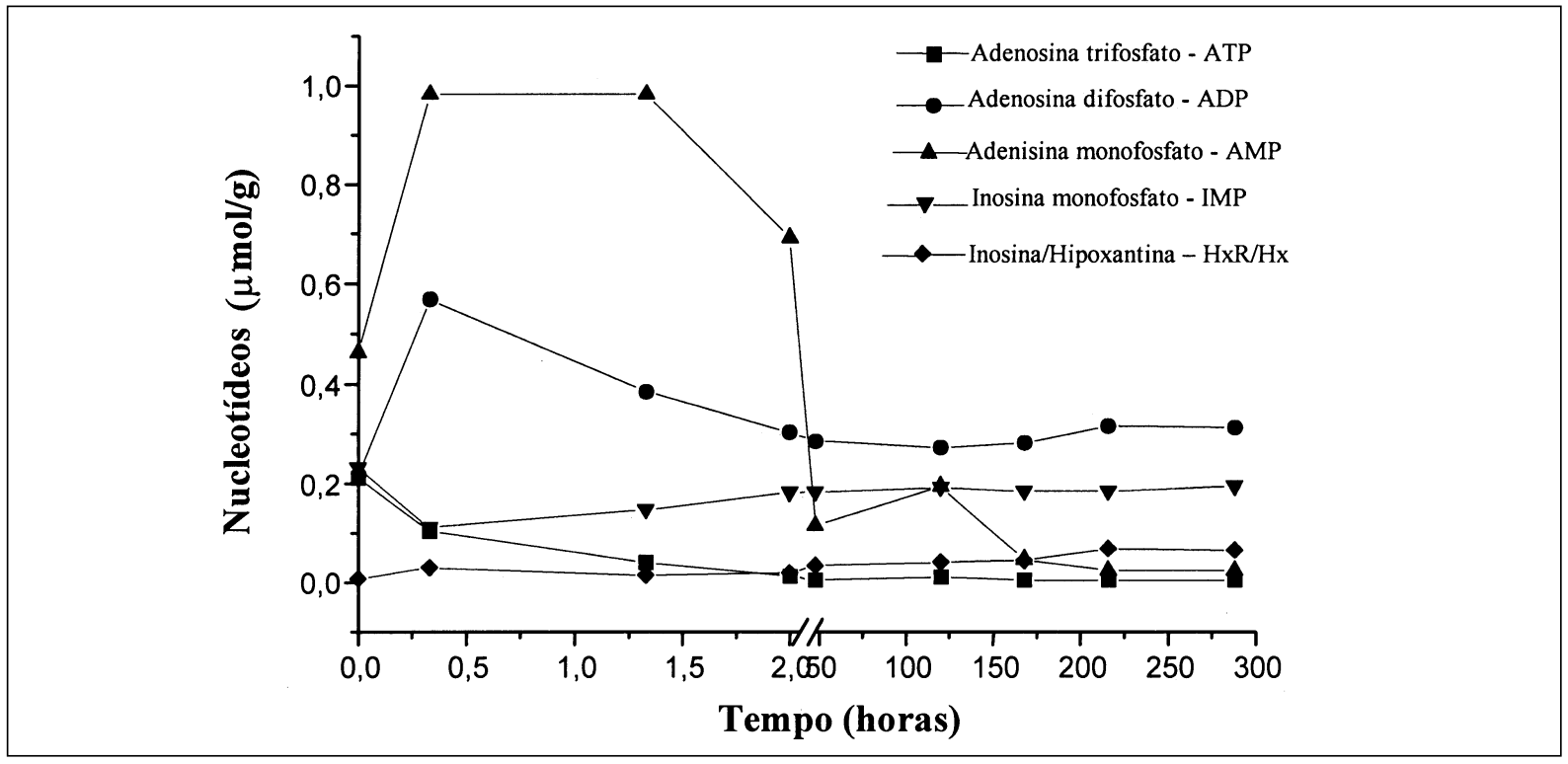

Figura 2 - Concentração de nucleotídeos do tambaqui, cultivado e estocado em gelo.

Ciência Rural, v.35, n.3, mai-jun, 2005. 
quantidades de inosina e hipoxantina, cuja expectativa seria de valores expressivos e crescentes. No entanto, detectaram-se pequenas quantidades durante os 12 dias de estocagem, apesar do valor de hipoxantina ser considerado como índice de determinação da qualidade de pescado de água doce (BEUCHAT, 1973).

$\mathrm{O}$ valor $\mathrm{K}$, relação entre os conteúdos de inosina, hipoxantina e os produtos de degradação do ATP no músculo de tambaqui, mostrou pequenas variações até 2 horas após a morte do pescado entre camadas de gelo. Em seguida, ocorreu um aumento progressivo, até um valor inferior a $12 \%$ ao final do período de estocagem com 12 dias. $\mathrm{O}$ valor $\mathrm{K}$ foi proporcional ao tempo de armazenamento (Figura 3).

TSUCHIMOTO et al. (1986) afirmaram que peixes de águas tropicais mostram menores mudanças no valor $\mathrm{k}$ do que peixes de águas temperadas. Nesta pesquisa, observou-se que o valor $\mathrm{K}$ encontrado no músculo do tambaqui até o $12^{\circ}$ dia de estocagem indicou alto nível de frescor, por se mostrarem menores que $12 \%$, valor bastante inferior aos $20 \%$ estabelecido por OKUMA et al. (1992) que classificaram o pescado que apresenta valor $\mathrm{K}$ inferior a $20 \%$ como altamente frescos aptos para o consumo cru, com valores entre $20-40 \%$ como frescos, devendo ser cozidos para consumo e pescados com valores acima de $40 \%$ como não frescos impróprios para o consumo humano. De acordo com ALMEIDA (1998), nesse mesmo período (com 12 dias de estocagem em gelo), o tambaqui foi considerado "qualidade especial" (classe A), segundo análise sensorial e valores das medidas físico-químicas de 6,45 e 10,73 para pH e N-BVT (Nitrogênio - Bases Voláteis Totais), respectivamente.

PEREZ et al. (2001) observaram que o valor K em híbridos de "cachama" cultivados, e armazenados a $0^{\circ} \mathrm{C}$, não ultrapassou $20 \%$ até o $14^{\circ}$ dia de estocagem em gelo. RYDER et al. (1984) estudaram o "jack mackerel" (Trachurus novaezelandiae) peixe marinho, estocado em gelo e observaram que ocorreu uma variação do valor $\mathrm{K}$ de $2 \%$ a $60 \%$. Alcançou $20 \%$ após 7 dias de estocagem em gelo, tempo em que a análise sensorial também indicou o final do tempo de vida útil. Estes autores concluíram que o valor $\mathrm{K}$ pode ser usado como indicador de qualidade para o consumo do peixe na forma de "sashimi" (peixe cru) e como índice de frescor durante o tempo de vida útil. HATULA et al. (1993) estudaram o "whitefish" (Coregonus wartmanni) que foi considerado aceitável, quando apresentou valor $\mathrm{K}$ acima de $80 \%$ e um valor mínimo de IMP de 1,0 mmol/g. VALLS et al. (1994) estudaram a sardinha (Sardinella aurita), inteira e eviscerada e conservada em gelo. Observaram que o valor $\mathrm{K}$ inicial foi semelhante para sardinha inteira e eviscerada e alcançaram valores finais de 70 e $73 \%$,

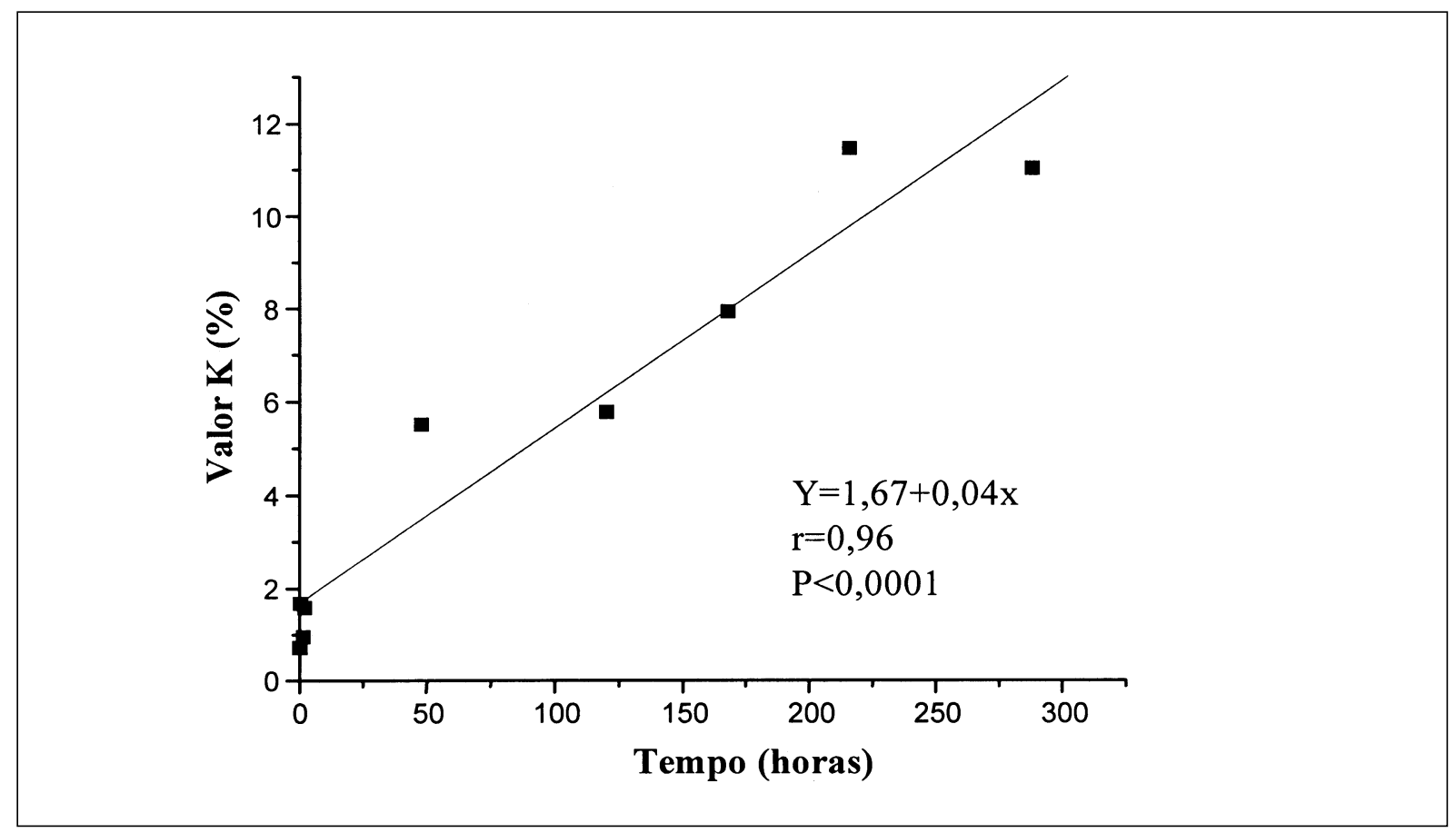

Figura 3 - Resultados do valor k, parâmetro utilizado para medir o frescor de peixe baseado nas alterações autolíticas, no músculo dorsal do tambaqui, cultivado e estocado em gelo.

Ciência Rural, v.35, n.3, mai-jun, 2005. 
respectivamente. A rejeição organoléptica para a sardinha inteira ocorreu no $8^{\circ}$ dia e correspondeu a um valor $\mathrm{K}$ de $54 \%$, enquanto que, para a eviscerada, a rejeição organoléptica ocorreu no $11^{\circ}$ dia e correspondeu a um valor $\mathrm{K}$ de $55 \%$.

\section{CONCLUSÕES}

O tambaqui cultivado e sacrificado entre camadas de gelo apresentou o fenômeno de "cold shock" e não rigor-mortis, por ter ocorrido contração muscular total quando $30 \%$ da concentração inicial de ATP ainda estava presente no músculo. A espécie apresentou no músculo baixos níveis de ATP e seus produtos de degradação e conseqüentemente, baixos valores $\mathrm{K}$ durante a estocagem. A determinação do valor $\mathrm{K}$, durante 12 dias de conservação em gelo, se revelou bom índice para avaliação do frescor.

\section{AGRADECIMENTOS}

À CAPESs pela concessão da bolsa de estudos e suporte financeiro. Ao Instituto Nacional de Pesquisas da Amazônia, (CPTA/LEEM), através do projeto CNPq/PTU/ $\mathrm{N}^{\circ} 680.190 / 96-9$.

\section{REFERÊNCIAS BIBLIOGRÁFICAS}

AMLACHER, E., Fish as Food. Berlin-Friedrichshagen, Germany. Institut fur Fischerei D. A. L., 1965. 2 v.

ALMEIDA, N,M. Alterações post-mortem em Colossoma macropomum Cuvier, 1818, procedentes da piscicultura, e conservados em gelo. 1998. 90 f. Dissertação (Mestrado em Ciência de Alimentos) - Curso de Pós-graduação em Ciência de Alimentos, Universidade do Amazonas.

BERAQUET, N.J.; LINDO, M.M.K. Transformações bioquímicas “post mortem” em pescado. São Paulo: Boletim ITAL, 1985.v.22, p. 169-192

BEUCHAT, L.R. Hipoxanthine measurement in assessing freshness of chilled channel catfish (Ictalurus punctatus). Journal Agriculture Food Chemical. v.21, n.3 p.453455,1973 .

BITO, M. et al. Studies on rigor mortis of fish - I. Difference in the mode of rigor mortis among some varieties of fish. By modified cuttingns methods. Bulletin Tokai Regional Fisheries Research Laboratory, v.109, p.89-96, 1983.

BOYD, N.S. et al. Effects of brain destruction on post harvest muscle metabolism in the fish kahawai (Arripis trutta). Journal of Food Science, v.49, p.177-179, 1984.

CURRAN, C.A. et al. Cold shock reaction in ice tropical fish. Journal Food Technology, v.21, p. 289-299, 1986.

FAO, World review of fisheries and aquaculture. Fisheries resources: trends in production, utilization and trade, 2000. Roma, Itália. 62p.
GRAEF, E.W. As espécies de peixes com potencial para criação no Amazonas. In: VAL. A.L.; HONCZARY, A. Criando peixe na Amazônia. 19.ed. Manaus : INPA 1995. p.29-43

HATTULA, T. et al. Freshness evalution in European whitefish (Coregonus wartmanni) during chill storage. Journal Food Science, v.58, p.1212-1236, 1993.

HUSS, H.H. El pescado fresco: su calidad y cambios de calidad. In: FAO. Manual de capicitación preparado por el programa de capacitación FAO/DANIDA en Tecnología pesquera y control de calidad. Roma : FAO. 1988. v.29.

JUNK, W.J. et al. The fish. In: JUNK, W.J. (ed). The central Amazon floodplain: ecology of a pulsing system. Ecological Studies, v.126, p.385-408, 1997.

MMA, IBAMA, CEPENE. Estatística da pesca - Brasil, grandes regiões e unidades da federação. Tamandaré, 2000. 95 p.

MMA, IBAMA,-SUPERINTENDÊNCIA ESTADUAL NO AMAZONAS. Relatório da operação defeso 1996/1997. Operação defeso, 1989 à 1997 SUPERINTENDÊNCIA ESTADUAL NO AMAZONAS-Manaus-Am. 1997. 19p.

NAMBURIDI, D.; GOPAKUMAR, K. Cold shock reaction in tropical fishes. Journal Food Science and Technology, v.25, p.89-91, 1988.

OKUMA, H. et al. Development of a system with double enzyme reactors for the determination of fish freshness. Analytica Chimica Acta, v.260, n.1, p.93-98, 1992.

PEREZ, M. et al. Efecto de la temperatura de almacenamiento sobre los cambios post-mortem y frescura en híbridos de Cachama (Colossoma macropomum $x$ Piaractus brachypomus) cultivados. Anales Venezolanos de Nutrición, v.14, n.2, p.53-59, 2001.

PARRY, R.W.H. et al. Cold shock in fish: its characteristics in bighead. Journal Food Science and Technology, v.22, p.637-642, 1987.

RABELO, A.M.A. Métodos físicos para análise do pescado, seminário sobre controle de qualidade na indústria de pescado. Santos, São Paulo : SBCTA/ITAL, 1988.

RYDER, J.M. et al. torage of New Zealand jack mackerel (Trachurus novaezelandiae) in ice: chemical, microbiological and sensory assessment. Journal of Food Science, v.49, p.1453-1477, 1984.

SAITO, T. et al. A new method for estimating the frehness of fish. Bulletin of Japanese Society of Scientific Fisheries, v.24, fasc.9, p.749-50, 1959.

SCHULTE, P.M. et al. Integrating metabolic partways in post exercise recovery of white muscle. Journal Experimental Biology, v.166, p.181-195, 1992.

TSUCHIMOTO, M. et al. The speed of lowering in freshness os fishes in several warters and the effect habitat temperature on the speed. Bulletin Japan. Society of Science Fisheries, v.52, p.1431-1441, 1986. 
VAL, A.L. et al. Effects of experimental anaemia on intraerythrocytic phosphate levels in rainbow trout, Oncorhynchus mykiss. Journal of Fish Biology, v.45, p.269-277, 1994 .

VALLS, J. et al. Determinación de amidas biógenas y nucleótidos por cromatogrfía líquida de alta eficiencia (HPLC) en muestras de sardinas (Sardinella aurita). Tercera consulta de expertos sobre tecnologia de produtos pesqueros en America Latina. Roma, Itália : FAO, 1994. 145p. (Inf de Pesca n.538. Suplemento, Mar).

ZAR. J.H. Biostatistical analysis. 3 ed. Upper Saddle River, NJ : Prentice Hall, 1996. 121p. 